\title{
TEM Sample Preparation of Heterogeneous Catalyst (Zeolite) Extrudates
}

\author{
Y.C. Wang ${ }^{1}$, Matt Kulzick ${ }^{1}$ and Eric Roth ${ }^{2}$ \\ ${ }^{1} \mathrm{BP}$, Naperville, Illinois, United States, ${ }^{2}$ Northwestern University, Evanston, Illinois, United States
}

The application of (scanning) transmission electron microscopy (S)TEM to the study of heterogeneous catalyst (zeolite-based) extrudates requires an electron-transparent thin section which preserves the structure and composition of both the zeolite and binder phases, in the as received condition. With a wellprepared sample the location and concentration distribution of different elements in the catalyst extrudate, critical to understanding and explaining catalyst performance (activity, selectivity, and stability), can be achieved. This talk will describe our procedure for obtaining these samples and detail the limits of detection for critical elements such as $\mathrm{Al}$ and $\mathrm{Si}$ that can be achieved on a modern analytical electron microscope.

In this report, we compare two TEM sample preparation techniques ultramicrotomy and focused ion beam sectioning. In order to examine heterogeneous catalysts using a typical medium voltage (80-200 keV) TEM, a sample thickness of approximately $100 \mathrm{~nm}$, or less is necessary. In the ultramicrotomy technique, the sample is first embedded in an appropriate polymer and a diamond knife is then used to slice thin sections. In this way it is possible to regulate the uniformity of the specimen thickness, typically 60-100 $\mathrm{nm}$. Focused ion beam (FIB) sectioning has also been widely used to prepare TEM or atom probe samples from heterogeneous catalysts. The FIB technique offers a site-specific sample preparation method, but we found it has inherent disadvantages for quantitation of highly localized elemental concentration differences inside the catalysts.

The FIB milling involves scanning a beam of ions across the sample. If the momentum of the ions as they strike the sample surface is high enough, atoms are sputtered away. Many times, the sputtered ions will redeposit on the sample surface. This may be mitigated at the fine milling stage with appropriate sample tilting and ion beam current. In this study, to minimize redeposition, we applied the focused ion milling at cryogenic temperature during the fine and final milling periods. A lift-out sample with $1 \mu \mathrm{m}$ thickness was prepared at room temperature using a standard FIB-SEM. Then, the sample was transferred into a cryo-FIB-SEM for additional thinning to prepare an electron-transparent section. The sample was maintained at $-135^{\circ} \mathrm{C}$ during this step to minimize ion beam damage. Figures $1 \mathrm{a}$ and $1 \mathrm{~b}$ show a typical FIB result with a zeolite-based catalyst.

For ultramicrotomy, catalyst extrudate samples were embedded in capsules with EMBed812 epoxy resin and then cured at $60^{\circ} \mathrm{C}$ for 48 hours. Blocks were carefully trimmed with a glass knife into a wedge-shaped blockface. This was placed on a Leica UC7 Ultramicrotome and a section thickness of approximately 60 nm was achieved using a diamond knife. Sections were collected on 200 mesh Au grids with a holey carbon membrane (Figures 1c and 1d).

Specimens were aluminosilicate zeolites bound with pure alumina binders. Thus, the concentration difference in silicon and aluminum between the zeolite and binder phase was stark and the bulk concentration of the silicon and aluminum content in both materials was known. The density difference between the two phases was also significant. Depending on the size of the zeolite and binder crystals, the sample thickness, and interface orientation, a gradient of concentration is expected between the two phases. If the aluminum- and silicon-oxide-based crystal bundle (slab-type) is tilted at $45^{\circ}$ (the worst condition) relative to the perpendicular crystal bundle (assuming $100 \mathrm{~nm}$ thick lamella), $40 \%$ shape factor might be introduced into the aluminum diffusion gradient measurement. 
Both cryogenic and room temperature prepared FIB zeolite catalyst TEM samples had around 3-20 wt\% Al concentration background in the Si domains, significantly higher than normal found in the bulk zeolite. This was caused by re-deposition during milling. The Al diffusion depth was $\sim 20 \%$ of the zeolite particle diameter (Figures $2 \mathrm{a}$ and $2 \mathrm{~b}$ ). Since the Cryo-FIB had much lower sputtering rate for the two phases, it was not able to evenly remove all the re-deposition. On the other hand, specimens prepared by ultramicrotomy had much lower $\mathrm{Al}$ concentrations in the zeolite domains. The $\mathrm{Al}$ concentrations in the zeolite domains of a 50:50 ZSM-5 (SAR=800)/Pural SCF (boehmite) sample were under 1\% Al and the $\mathrm{Al}$ diffusion depth was only a few percent of the zeolite diameter. (Figures $2 \mathrm{c}$ and $2 \mathrm{~d}$ ). This illustrates the advantage of ultramicrotomy for preparing zeolite catalyst specimens for measuring the aluminum concentration in the zeolite phase and examining the binder/zeolite interface [2].
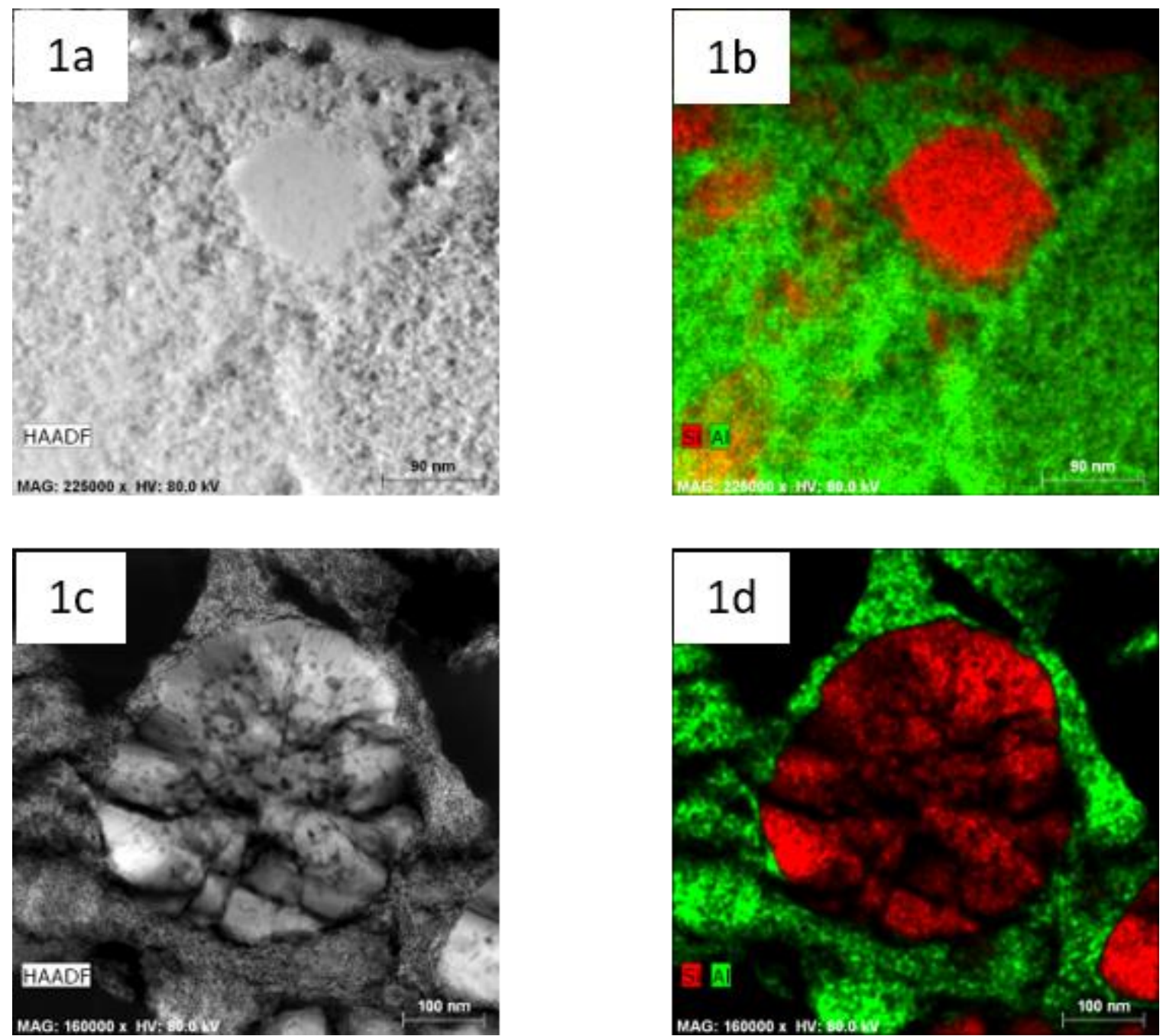

Figure 1. STEM image (1a) and STEM-EDS map (1b) of a FIB prepared cross sections of large Si domains are on the same approximate scale as ZSM5 crystal bundles and STEM image (1c) and STEMEDS map (1d) of a microtome prepared cross sections of mixed-size Si domains are on the same approximate scale as ZSM5 crystal bundles. 

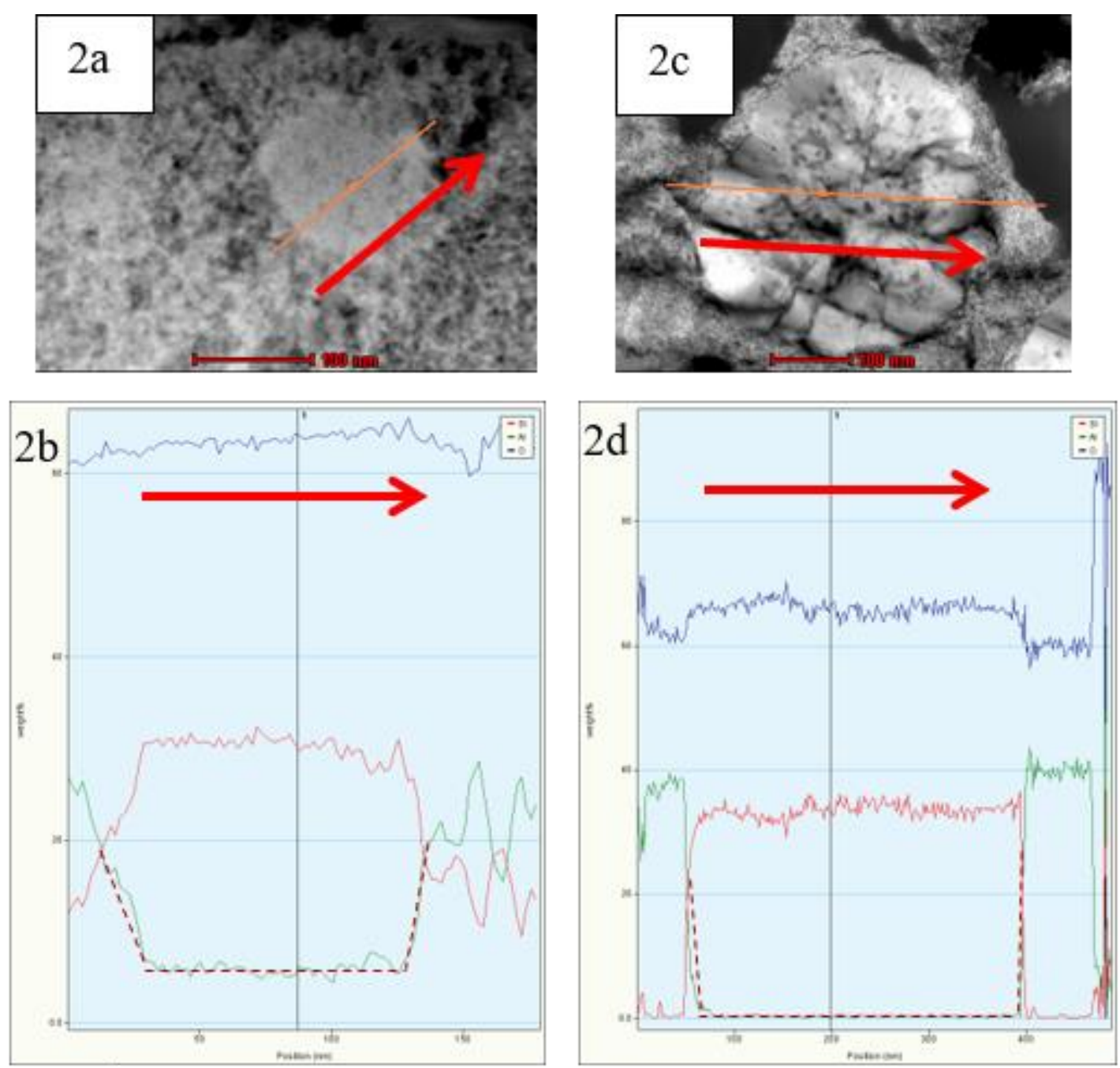

\section{To indicate the estimated $\mathrm{Al}$ diffusion gradient}

Figure 2. A STEM reference image (2a) of an EDS lineprofile (2b) indicates Al concentration background is less than $10 \mathrm{wt} \%$ across the ZSM5 particle and a STEM reference image (2c) of an EDS lineprofile (2d) indicates $\mathrm{Al}$ concentration is less than $1.0 \mathrm{wt} \%$ across the ZSM5 particle.

\section{References}

1. R. Haswell, D.W. McComb, W. Smith, Journal of Microscopy 211, Pt. 2, 161-166 (2003).

2. The authors gratefully acknowledge Tseng-Ming Chou of Stevens Institute of Technology for providing fine and final cryo-FIB-SEM milling of the FIB prepared TEM samples. 